\title{
Anleitung zur Betonung lateinischer Wörter
}

1. Zweisilbige Wörter werden auf der vorletzten Silbe hetont.

2. Mehrsilbige Wörter werden auf der drittletzten Silbe betont, außer wenn die vorletzte Silbe lang ist.

3. Als lang gelten alle Silben, auf deren Vokal mehrere Konsonanten folgen.

\section{Das griechische Alphabet}

\begin{tabular}{|c|c|c|c|c|}
\hline \multicolumn{2}{|c|}{$\begin{array}{l}\text { zrobe kleine } \\
\text { Buchstabeu }\end{array}$} & \multicolumn{2}{|c|}{ Name } & \multirow{2}{*}{$\begin{array}{c}\text { Laute } \\
\mathbf{a}\end{array}$} \\
\hline$A$ & $u$ & álpha & 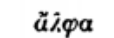 & \\
\hline$B$ & $\beta$ & bēta & $\beta \tilde{\eta} \tau \alpha$ & b \\
\hline$I$ & $\gamma$ & gámma & $\gamma \dot{\alpha} \mu \mu a$ & $\mathrm{~g}$ \\
\hline 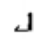 & $\delta$ & délta & $\delta \varepsilon \dot{\lambda} \tau \alpha$ & d \\
\hline$E$ & $\varepsilon$ & ě-psílon & $\tilde{\varepsilon} \cdot \psi t \lambda .0 v$ & $\check{\theta}$ \\
\hline$Z$ & $\zeta$ & dséta. & $\zeta \bar{\eta} \tau \alpha$ & ds \\
\hline$I I$ & $\eta$ & êta & $\bar{\eta} \tau \alpha$ & $\overline{\mathrm{e}}$ \\
\hline$\Theta$ & $\vartheta$ & theta & $\vartheta \tilde{\eta} \tau \alpha$ & th \\
\hline$I$ & ! & iōta & $i \tilde{\omega} \tau a$ & $\mathbf{i}$ \\
\hline$K$ & $x$ & káppa & 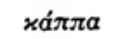 & $\mathbf{k}$ \\
\hline . & $\lambda$ & lámbda & $\lambda \dot{\alpha} \mu \beta \delta \alpha$ & 1 \\
\hline$M$ & $\mu$ & $m \bar{y}$ & $\mu \bar{v}$ & $\mathbf{m}$ \\
\hline$N$ & $v$ & $\mathbf{n \overline { y }}$ & $v \overline{0}$ & $\mathbf{n}$ \\
\hline$\Xi$ & $\xi$ & $x^{i}$ & $\xi i$ & $x$ \\
\hline$O$ & 0 & ŏ-mikrón & 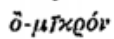 & ǒ \\
\hline$\Pi$ & $\pi$ & pî̀ & $\pi i$ & p \\
\hline$P$ & $\varrho$ & rhō & 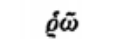 & $\mathbf{r}$ \\
\hline$\Sigma$ & $\left.\sigma^{*}\right)$ & sigma & $\sigma \bar{\imath} \gamma \mu \alpha$ & $\mathbf{s}$ \\
\hline$T$ & $\tau$ & $\operatorname{tau}$ & $\tau \alpha \tilde{i}$ & $t$ \\
\hline$Y$ & $v$ & y-psílón & $\dot{v}-\varphi \bar{T} \lambda o ́ v$ & $\overline{\mathbf{u}}$ \\
\hline$\Phi$ & $\varphi$ & phì & $\varphi \bar{i}$ & ph (f) \\
\hline$X$ & $\chi$ & chi & $\chi^{\bar{i}}$ & ch \\
\hline$\Psi$ & $\psi$ & psî̀ & $\varphi \bar{\imath}$ & ps \\
\hline$\Omega$ & $\omega$ & ō-mĕga & $\omega-\mu \varepsilon \gamma \alpha \alpha$ & $\overline{0}$ \\
\hline
\end{tabular}

*) $\sigma$ im An- und Inlaut; $\varsigma$ im Auslaut. 